\title{
A Comparative Analysis of Lower Extremity Joint Pain and the Risk of Falling in Elderly Patients
}

\author{
Xia Huang, Guiyan Wen* \\ Department of Rheumatology, The First Affiliated Hospital of Jinan University, Guangdong, Guangzhou, China \\ Email address: \\ 15915772676@163.com (Xia Huang),wengy78@126.com (Guiyan Wen) \\ ${ }^{*}$ Corresponding author
}

To cite this article:

Xia Huang, Guiyan Wen. A Comparative Analysis of Lower Extremity Joint Pain and the Risk of Falling in Elderly Patients. American Journal of Nursing Science. Vol. 10, No. 1, 2021, pp. 13-16. doi: 10.11648/j.ajns.20211001.13

Received: December 2, 2020; Accepted: December 14, 2020; Published: January 12, 2021

\begin{abstract}
Background: It is well known that chronic lower limb joint pain in elderly patients is closely related to the risk of falls. Understanding the relationship between pain and the risk of falling could help prevent it. Objective: To investigate the correlation between the location and degree of lower extremity joint pain and the risk of falling among newly admitted elderly patients in the Department of Rheumatology, to search for personalized care countermeasures to prevent elderly patients from falling, and to improve the safety of elderly inpatients in the Department of Rheumatology. Method: A total of 214 elderly patients admitted to the Department of Rheumatology with different lower extremity joint pains were evaluated for the location and degree of their joint pain and their risk of falling. After which, an analysis was done to determine the correlation between the location and degree of lower extremity joint pain and the risk of falling and provide targeted nursing countermeasures to prevent falling. Results: By grading the patients' pain levels, assessing their risk of falling, and taking corresponding nursing countermeasures to prevent falling, 214 elderly patients with different lower extremity joint pains did not fall during their stay in the hospital. Conclusion: Through pain assessment and fall risk assessment and analysis, targeted measures can be taken to effectively prevent falls and reduce the occurrence of accidental injuries among elderly patients.
\end{abstract}

Keywords: Joint Pains, Elderly Patients, Pain Level Grading, Fall Risk Assessment

\section{Introduction}

Falls and fall-related injuries are common and a serious problem in older people. People over 65 years of age have the highest risk of falling, with an estimated one-third of older people falling at least once a year [1].

The International Working Group on the Prevention of Falls in Older Persons defines a fall as an unintentional fall to the ground or a lower level. However, it does not include falls caused by violence, loss of consciousness, hemiplegia, or seizures. [2] Falls, as one of the most common disease-causing risks for older adults, cause psychological trauma, loss of motor function and self-care, and significant financial losses. [3] Falls are the most common type of negative incidents reported in hospitals and the second leading cause of harm to inpatients. [4] Nowadays, foreign and Chinese scholars have gradually recognized that injuries such as falls can be predicted, prevented, and controlled. [5] An assessment of fall-related risk factors is crucial for fall prevention. Several domestic and international studies [6-8] have shown that the assessment of fall-related risk factors and the development and implementation of preventive measures can positively impact the prevention of falling among the elderly. As older adults age, they experience decreased muscle strength and joint mobility, especially the impairment of balance and gait, affecting their ability to move and resulting in falls.[9] Studies have shown that falls due to pain and muscle weakness caused by knee and ankle osteoarthritis account for $75 \%$ of physical falls among older adults. [10] A study conducted by Guo Yan-mei et al. showed that elderly patients with knee osteoarthritis (KOA) and unilateral knee pain are at higher risk of falling due to the decrease in their dynamic balance function, compared to those without knee pain. In contrast, elderly patients with KOA and bilateral knee pain are at a higher risk of falling due to the simultaneous decrease in their static and dynamic balance functions. [11] Pain, one of the most common clinical symptoms, is a complex, subjective sensation and a 
clinical problem that has received much attention recently. Pain has a great impact on the ability of patients with musculoskeletal disorders to take care of themselves in their daily lives, especially for women and elderly patients. [12] Joint pain is a common symptom of lower extremity arthritis and disease; pain is also a risk factor for falls. Therefore, assessing the location and extent of pain and assessing the risk of falling in elderly patients with different lower extremity joint pains can effectively identify the fall risks among such elderly patients and reduce blindness and passivity in fall prevention.

\section{Clinical Data and Methods}

\subsection{Clinical Data}

The 214 medical cases of elderly patients aged $\geq 65$ years old with lower extremity arthritis used for this study were taken from the Department of Rheumatology and Immunology of the First Affiliated Hospital of Jinan University. They were patients from March 2017 to March 2019 and included 123 males and 91 females. Among the patients, the oldest was 96 years old and the youngest was 65 years old, with the mean age being 72.5 years old. All cases met the American College of Rheumatology's revised 1982 diagnostic criteria for gouty arthritis and rheumatoid arthritis.

\subsection{Method}

The 214 elderly patients complaining of lower extremity joint pain were divided into eight groups corresponding to eight different kinds of lower extremity joint pain. Nurses used the Verbal Rating Scale (VRS) for pain assessment and the Fall Risk Assessment Scale for the Elderly (FRASE) for fall risk assessment to compare the pain severity and fall risks of the eight groups. The VRS was the earliest scale used in pain research, with each grade describing pain severity. Pain is classified as grade 0 -III on the VRS score. VRS scale is considered as an important method for pain assessment after orthopaedic surgery. [13] The FRASE [14] measures the risk of falls among elderly inpatients and is correlated with STRATIFY. The eight items included in the scale are gender, sensory function, current diagnosis, age, history of falls, gait, physical activity status, and medication usage. A total score of 3 to 8 is classified as having a low risk of falling; 9 to 12 , having a moderate risk of falling; and 13 or more, having a high risk of falling.

\section{Results}

The relationship between pain assessment and risk of falls in the eight groups of elderly patients was as follows: $75.86 \%$ of those in the single metatarsal joint pain group had a pain level of II to III and $31.04 \%$ of them had a high risk of falling, with high risk of falling accounting for $40.92 \%$ of the group's total; $84.62 \%$ of those in the two metatarsal joints pain group had a pain level of II to III and $61.54 \%$ of them had a high risk of falling, with high risk of falling accounting for $72.73 \%$ of the group's total; $75.00 \%$ of those in the single ankle joint pain group had a pain level of II to III and $16.67 \%$ of them had a high risk of falling, with high risk of falling accounting for $22.23 \%$ of the group's total; $68.42 \%$ of those in the two ankle joints pain group had a pain level of II to III and $36.84 \%$ of them had a high risk of falling, with high risk of falling accounting for $53.84 \%$ of the group's total; $60 \%$ of those in the single knee joint pain group had a pain level of II to III and $13.33 \%$ of them had a high risk of falling, with high risk of falling accounting for $22.22 \%$ of the group's total; $61.54 \%$ of those in the two knee joints pain group had a pain level of II to III and $39.08 \%$ of them had a high risk of falling, with high risk of falling accounting for $61.88 \%$ of the group's total; $80.00 \%$ of those in the hip joint pain group had a pain level of II to III and $60.00 \%$ of them had a high risk of falling, with high risk of falling accounting for $75.00 \%$ of the group's total; $73.91 \%$ of those in the multiple joints pain group had a pain level of II to III and high risk of falling $56.92 \%$ of them had a high risk of falling, with high risk of falling accounting for $77.00 \%$ of the group's total. The differences between the eight groups were statistically significant $(\mathrm{P}<0.05)$, and the detailed results are shown in Table 1 . When there is a high level of joint pain, the risk of falling is also higher. The risk of falling for those with multiple lower extremity joint pain is higher than for those with single lower extremity joint pain. The risk of falling for those with proximal lower extremity joint pain is higher than that of those with distal lower extremity joint pain.

Table 1. Comparison of the pain levels and risk of falling among the eight groups.

\begin{tabular}{|c|c|c|c|c|c|c|c|c|c|c|}
\hline \multirow{3}{*}{ Groups } & \multirow{3}{*}{$\begin{array}{l}\text { Number } \\
\text { of } \\
\text { medical } \\
\text { cases } n\end{array}$} & \multicolumn{9}{|c|}{ VRS Pain Rating / FRASE Fall Risk } \\
\hline & & \multicolumn{3}{|c|}{ VRS Level I } & \multicolumn{3}{|c|}{ VRS Level II } & \multicolumn{3}{|c|}{ VRS Level III } \\
\hline & & $\begin{array}{l}\text { Low } \\
\text { risk }\end{array}$ & $\begin{array}{l}\text { Medium } \\
\text { risk }\end{array}$ & $\begin{array}{l}\text { High } \\
\text { risk }\end{array}$ & $\begin{array}{l}\text { Low } \\
\text { risk }\end{array}$ & $\begin{array}{l}\text { Medium } \\
\text { risk }\end{array}$ & $\begin{array}{l}\text { High } \\
\text { risk }\end{array}$ & $\begin{array}{l}\text { Low } \\
\text { risk }\end{array}$ & $\begin{array}{l}\text { Medium } \\
\text { risk }\end{array}$ & $\begin{array}{l}\text { High } \\
\text { risk }\end{array}$ \\
\hline Single metatarsal joint pain group & 39 & $24.14 \%$ & 0 & 0 & 0 & $44.82 . \%$ & $6.90 \%$ & 0 & 0 & $24.14 \%$ \\
\hline Two metatarsal joints pain group & 23 & $15.38 \%$ & 0 & 0 & 0 & $23.08 \%$ & 0 & 0 & 0 & $61.54 \%$ \\
\hline Single ankle joint pain group & 22 & $25.00 \%$ & $25.00 \%$ & 0 & $7.82 \%$ & $25.51 \%$ & 0 & 0 & 0 & $16.67 \%$ \\
\hline Two ankle joints pain group & 27 & $21.05 \%$ & $10.53 \%$ & 0 & 0 & $31.58 \%$ & $15.79 \%$ & 0 & 0 & $21.05 \%$ \\
\hline Single Knee joint pain Group & 23 & $33.33 \%$ & $6.67 \%$ & 0 & 0 & $46.67 \%$ & & 0 & 0 & $13.33 \%$ \\
\hline Two Knee joints pain Group & 34 & $23.08 \%$ & $15.38 \%$ & 0 & 0 & $22.39 \%$ & $23.77 \%$ & 0 & 0 & $15.38 \%$ \\
\hline Hip joint pain group & 11 & 0 & $20.00 \% \%$ & 0 & 0 & $20.00 \%$ & 0 & 0 & 0 & $60.00 \%$ \\
\hline Multiple joints pain group (three or more joints) & 34 & 0 & $21.74 \%$ & $4.35 \%$ & 0 & $21.74 \%$ & $13.04 \%$ & 0 & 0 & $39.13 \%$ \\
\hline
\end{tabular}

Eight groups compared, * $\mathrm{p}<0.05$; VRS:Verbal Rating Scale, FRASE:Fall Risk Assessment Scale for the Elderly. 
The VRS pain scale was used to assess each group of elderly patients with lower extremity joint pain during their first nursing assessment at the hospital and, using the FRASE, it was found that the risk of falling was related to pain in the lower extremity joints. In relation to the location of the lower extremity joint pain, the following are ranked from the highest to the lowest risk of falling: multiple lower extremity joints, hip joint, two knee joints, two ankle joints, two metatarsal joints, one metatarsal joint, single ankle joint, and single knee joint. In relation to pain levels, the following are ranked from the highest to the lowest risk of falling: VRS Level III, VRS Level II, and VRS Level I. The differences between the compared groups were statistically significant $(\mathrm{P}<0.05)$, and the detailed results are shown in Tables 2 and 3.

Table 2. Comparison of the high risk of falling in the multiple lower extremity joint pain group versus the single knee joint pain group among 214 patients.

\begin{tabular}{lll}
\hline Groups & Multiple lower extremity joints & Single knee joint \\
\hline Number of medical cases (n) & 34 & 23 \\
Pain level 2 to 3 & $73.91 \%$ & $60.00 \%$ \\
High risk of falling percentage & $77.00 \%$ & $22.22 \%$ \\
\hline
\end{tabular}

Compared to the single knee joint pain group, $* \mathrm{P}<0.05$.

Table 3. Comparison of risk of falling for different pain levels among 214 patients

\begin{tabular}{llll}
\hline Groups & VRS Level I & VRS Level II & VRS Level III \\
\hline Number of cases (n) & 44 & 55 & 45 \\
Low risk of falling percentage & $91.74 \%$ & $6.40 \%$ & 0 \\
Medium risk of falling percentage & $7.20 \%$ & $68.15 \%$ & 0 \\
High risk of falling percentage & $1.06 \%$ & $25.45 \%$ & $100 \%$. \\
\hline
\end{tabular}

$* \mathrm{P}<0.05$ for high risk of falling in VRS Level I versus VRS Level III.

\section{Discussion}

Falls and fall-related injuries are common and a serious problem in older people. People over 65 years of age have the highest risk of falling, with an estimated one-third of older people in the hospital falling at least once a year. [1] Among our study, the oldest was 96 years old and the youngest was 65 years old, with the mean age being 72.5 years old, which similar to literature reports. The rate of fall-related injuries also increases with age. Most fall-related injuries are minor, such as bruising, abrasions, lacerations, strains and sprains, but can still cause significant pain and discomfort. However, some falls can have serious long-term consequences, including fall-related fractures and head injuries. Therefore, early recognition and assessment of fall-related risk factors and early intervention to reduce adverse outcomes are the fundamental objectives of our research.

An international consensus statement defined a fall as "an unexpected event in which the participant comes to rest on the ground, floor or lower level". [15]

These risk factors can be broadly categorised as either intrinsic or extrinsic. Intrinsic fall-related risk factors include advanced age, history of previous falls, muscle weakness, gait and balance problems, poor vision, and chronic diseases such as arthritis, diabetes, stroke, Parkinson's, dementia and incontinence. Extrinsic fall-related risk factors include environmental factors such as lack of hand rails, poor lighting, slippery or uneven surfaces, use of walking aids and poor footwear. It is estimated that around $15 \%$ of falls result from a major external event that would cause most people to fall. A similar percentage of falls result from a single identifiable event such as syncope (fainting). However, most result from multiple interacting factors (e.g. a person has balance problems, poor vision and slips on an uneven surface which results in a fall) [16]. Generally, the more risk factors a person has, the greater their chances are of having a fall.

There are many factors associated with the falling of elderly inpatients, and this may be the result of multiple factors working together. [17] Gouty arthritis and rheumatoid arthritis, which are both diseases related to the immune system and motor system, are factors related to falling among the elderly. There are also other fall-related factors among this group of elderly patients, such as physiological factors (e.g. gender and age), disease-related factors (e.g. diabetes, hypertension, coronary heart disease, stroke, and fever), medication factors (e.g. taking antihypertensive, hypoglycemic, or analgesic medications), the use of assistive devices (e.g. wheelchairs and crutches), environmental factors (e.g. hospital ramps), and the deterioration of sensory functions (e.g. hearing and visual impairment); all these factors increase their risk of falling. Clinical nurses assess the pain level and fall risk of elderly patients, prompting clinicians, nurses, and patients and their families to take active control of the patients' pain, pay attention to the patient's fall risk, and provide targeted measures to prevent falling, which can all effectively prevent falling from occurring.

Osteoarthritis (OA) is a common clinical degenerative disease, while knee osteoarthritis (KOA) causes dysfunction in the daily life of the elderly due to its pain, thus increasing the risk of many diseases. Whether it is OA or KOA, it is a high-scoring disease in the elderly manifested by varying degrees of pain caused by inflammatory activities of lower limb joints. Among them, sudden unbearable pain is the inherent reason that leads to the increased risk of fall in the elderly. Pain is classified as grade I-III on the VRS score. VRS scale is considered as an important method for pain assessment after orthopaedic surgery [13] The elderly patients in our study are all at risk of falling, but the degree to which 
they are at risk varies by lower extremity joint pain location and pain levels. They experience pain in different lower extremity joints, which affects their motor system functions and decreases muscle strength and joint flexibility, resulting in slow movements, staggering gait, and increased risk of falling. Based on the study, it is clear that elderly patients who experience pain in multiple lower extremity joints have a higher risk of falling than those who experience pain in a single joint; the higher the level of pain they experience, the higher their risk of falling.

\section{Conclusion}

The risk of fall was related to the pain site and degree of lower limb joints, and the risk of fall related to pain lower limb joints was from high to low: multiple lower limb joints, hip joint, double knee joint, double ankle joint, double metatarsal joint, single metatarsal joint, single ankle joint, and single knee joint. Other foreign reports have reached similar conclusions. [1, 12, 17] The fall risk associated with pain was from high to low: VRSIII, VRSII, VRSI. Therefore, we call attention to the number and degree of lower extremity joint pain in elderly inpatients.

\section{Abbreviations}

KOA: knee osteoarthritis

OA: Osteoarthritis

VRS: Verbal Rating Scale

FRASE: Fall Risk Assessment Scale for the Elderly

\section{Acknowledgements}

I would like to thank my colleagues in the Department of Gastroenterology and Rheumatology of the First Affiliated Hospital of Jinan University for their assistance in the publication of this paper. In addition, I would also like to thank different colleagues for their help in collecting data and completing various evaluations in daily work.

\section{References}

[1] Meyer M, Constancias F, Vogel T, Kaltenbach G, Schmitt E: Gait Disorder among Elderly People, Psychomotor Disadaptation Syndrome: Post-Fall Syndrome, Risk Factors and Follow-Up - A Cohort Study of 70 Patients. Gerontology 2020, 30: 1-8.

[2] Tan Zhaohui and Yu Pulin. The risk of falls and fractures in the elderly and their prevention. Chinese Journal of Practical Internal Medicine, 2011, 31 (1): 28-30.

[3] $\mathrm{Xu}$ Jianhong. The latest nursing evidence on preventing inpatients from falling [J]. Journal of Practical Nursing. 2001, 17 (17): 38-39.
[4] Turner K, Staggs V, Potter C. Fall prevention implementation strategies in use at 60 United States hospitals: a descriptive study. BMJ Quality \& Safety 2020, 29: 1000-1007.

[5] Harbison AJ, Prabhu S. Causation and Treatment Algorithms for Elderly Patients who have Fallen in the Twin Tiers. Cureus. 2019, 11 (12): e6513.

[6] Deng Fei-Fei and Gan Xiuni, Meta-analysis of the effect of exercise and multifactorial assessment and intervention on fall prevention in older adults. Chinese Journal of Gerontology, 2011, 31 (5): 735-738.

[7] Xia Qinghua and Jiang Yu. Research on the effectivity and needs of interventions on the risk factors of the elderly falling at home. China Health Education, 2010, 26 (8): 607-608.

[8] Alacculloch P. A., Gardner T., and Bonner A. Comprehensive fall prevention programs across settings A review of the literature. Geriatric Nursing, 2007, 28 (5): 306-311.

[9] Zhao Liqun and Wan Qiaoqin. Development of a fall risk assessment tool for the elderly. Chinese Nursing Management, 2012, 12 (11): 51-54.

[10] Laura A. W., Drryl G. T., Albert B. S., et al. Age and gender differ-ence in peak lower extremity joint torquce and ranges of motion used during single-step balance recoery front a forward fall J Biomech, 2001, 34 (1): 67-75.

[11] Guo Yanmei, Huang Peng, Chen Wei, Jiao Weiguo, and Weng Changshui, Comparative analysis of balance function and risk of falling in elderly patients with osteoarthritis of one or both knees. Theory and Practice of Rehabilitation in China. 2012, 18 (1): 1 .

[12] Chen Jing, Wu Qing, and Yang Lei, Impact of pain in musculoskeletal disorders on patients' ability to perform daily activities. Environmental and Occupational Medicine. 2003, 12 (6): 433.

[13] Bech RD, Lauritsen J, Ovesen O, Overgaard S. The Verbal Rating Scale Is Reliable for Assessment of Postoperative Pain in Hip Fracture Patients. Pain Res Treat. 2015, 7: 676212.

[14] Ann L. Hendrich, Patricias. Bender, and Allen Nyhuis. Validation of the Hendrieh II Fall Risk Model: A Large Concurrent Case/Control Study of HosPitalized Patients. APPlied nursing researeh, 2003, 16 (1): 9-21.

[15] Lamb SE, Jørstad-Stein EC, Hauer K, Becker C, Prevention of Falls Network Europe and Outcomes Consensus Group. Development of a common outcome data set for fall injury prevention trials: the Prevention of Falls Network Europe consensus. Journal of the American Geriatrics Society 2005; 53 (9): 1618-22.

[16] Campbell AJ, Robertson MC. Implementation of multifactorial interventions for fall and fracture prevention. Age and Ageing 2006, 35 (2): 60-64.

[17] Rong Lan, Zhu Ping, and Yu Xiaoping. Research progress on the factors associated with falls in hospitalized elderly patients. Shanghai Nursing 2018, 10 (2): 75-80. 